\title{
Clinical Effect of Electroacupuncture on Lung Injury Patients Caused by Severe Acute Pancreatitis
}

\author{
Li Li, ${ }^{1}$ Jianbo Yu, ${ }^{2}$ Rui Mu, ${ }^{2}$ and Shuan Dong ${ }^{2}$ \\ ${ }^{1}$ Department of Anesthesiology, Tianjin Central Hospital of Gynecology Obstetrics, Tianjin 300100, China \\ ${ }^{2}$ Department of Anesthesiology, Tianjin Nankai Hospital, Tianjin 300100, China \\ Correspondence should be addressed to Jianbo Yu; yujianbo11@126.com
}

Received 6 July 2016; Revised 27 October 2016; Accepted 6 March 2017; Published 29 June 2017

Academic Editor: Tse H. Huang

Copyright (c) $2017 \mathrm{Li} \mathrm{Li}$ et al. This is an open access article distributed under the Creative Commons Attribution License, which permits unrestricted use, distribution, and reproduction in any medium, provided the original work is properly cited.

\begin{abstract}
This study aimed to investigate the effects of electroacupuncture at the Lieque, Chize, and Zusanli points in patients with lung injury caused by severe acute pancreatitis. Patients with acute respiratory distress syndrome (ARDS) induced by severe acute pancreatitis (SAP) were randomly divided into three groups based on the treatment: conventional therapy alone (group A), electroacupuncture of nonacupoints with conventional therapy (group B), and electroacupuncture at the Lieque (LU7), Chize (LU5), and Zusanli (ST36) points (group C) once a day for 5 days. Arterial blood samples were obtained for blood gas analysis before electroacupuncture (T1) and $3(T 2)$ and 5 (T3) days after electroacupuncture. The oxygenation index was significantly higher in all groups at $T 2$ and $T 3$ than that at $T 1$, while the APACHE-II scores were decreased significantly. The expression of TNF- $\alpha$ was significantly decreased and the IL-10 was significantly increased in all groups at $T 3$. The oxygenation index at $T 2$ and $T 3$ was significantly higher in group C than that in group B. Electroacupuncture at Lieque, Chize, and Zusanli can lessen the lung injury induced by SAP, and the mechanism may be related to the decreased TNF- $\alpha$ and increased IL-10 value. Clinical Registration Number is ChiCTR-ICR-15006850.
\end{abstract}

\section{Introduction}

Severe acute pancreatitis (SAP) is a common acute syndrome that is characterized by pancreatic digestion by its own enzymes and necrosis. SAP is difficult to treat with conservative measures and therefore requires surgery. It can often cause systemic inflammatory response syndrome (SIRS) [1] and the associated mortality rate ranges from 25 to $30 \%$ [26]. Related researches have illustrated that lung is the earliest and most easily damaged organ in SAP. Acute pancreatitisassociated lung injury (APALI) is the most common early complication, and acute lung injury is the main cause of high mortality in SAP [7]. Unfortunately, none of the currently available drugs are effective in treating SAP [8]. Therefore, determining how to effectively regulate the inflammatory reactions in SAP and prevent the consequent lung injury is of great clinical significance.

Within the concepts of traditional Chinese medicine (TCM), maintenance of health and normalization of body function stems from a balance of "yin" and "yang" and free flow of energy (Qi) along various meridians in the body. Previous studies have suggested that acupuncture, a technique which facilitates free flow of Qi, can alleviate dyspnoeic symptoms, reduce postexercise bronchoconstriction, and improve exercise capacity. Studies have demonstrated that electroacupuncture can regulate the release of inflammatory factors and immune stress response [9]. Electroacupuncture at the Zusanli (ST36) point in rats has been shown to inhibit the release of inflammatory mediators and reduce endotoxininduced acute kidney injury [10]. Electroacupuncture at the Zusanli point has a bidirectional benign regulatory role in the pituitary-adrenal cortex system function and improves the immune ability [11]. The Lieque (LU7) point is an important acupuncture point on the lung meridian, which is clinically used in the treatment of respiratory diseases. Studies have shown that electroacupuncture at the Lieque point could improve the symptoms and pulmonary functions of patients with asthma or chronic obstructive pulmonary disease by regulating the autonomic nervous system, promoting the release of endogenous peptide at the hypothalamic level, 
and inhibiting the release of inflammatory cytokines [12-14]. Chize (LU5) belongs to the lung meridian, which also participates in the regulation of lung functions according to the theory of traditional Chinese medicine (TCM). Pairing of the viscera and bowels is an important theory, which provides guidance to TCM clinical practice. Recently, researchers have performed many studies on the theory that the lung and large intestine are exterior-interiorly related, which is a different point of view to that of previous literature; recent clinical studies and experimental studies have enforced the theoretical connotation of the statement. Studies have also shown that electroacupuncture stimulation at Chize could effectively inhibit the effect of enteritis on the lungs, decrease the level of tumor necrosis factor- (TNF-) $\alpha$, and reduce the ulcerative colitis-induced lung injury significantly [15]. During clinical treatment, different acupuncture points that are compatible with medicines are selected for treating diseases. The present study intended to apply electroacupuncture at the Lieque, Chize, and Zusanli points to determine whether this treatment could improve the degree of lung injury caused by SAP.

\section{Materials and Methods}

The present research was approved by the Medical Ethics Committee of Nankai Hospital, Tianjin City (number tjnk20130618), Clinical Registration Number: ChiCTR-ICR15006850. Informed consent was obtained from the patients and/or their families. Total of 60 patients who were newly diagnosed as acute respiratory distress syndrome (ARDS) induced by SAP were recruited. Exclusion criteria included previous bronchial and pulmonary diseases, recent hormone use, recent immunosuppressive therapy, and recent acupuncture treatment. ARDS was diagnosed according to the ARDS diagnostic criteria chaired and revised by the European critical disease association in Berlin of Germany in 2011 [16]. Patients were randomly divided into three groups $(n=20)$ using a computer-generated random number table. All the acupuncture treatments were completed by the same physician with 10 years of clinical experience and professional operational skills. In group A, only conventional therapy was conducted without acupuncture treatment. In group B, electroacupuncture of nonacupoints (one centimeter away from Lieque, Chize, and Zusanli points but different from the traditional Chinese medicine "Jing Luo") was conducted based on conventional therapy with the same acupuncture parameters as those in group C. In group C, electroacupuncture was carried out at the Lieque point (LU7; on the radial aspect of the forearm, between the tendons of the abductor pollicis longus and the extensor pollicis brevis muscles, in the groove for the abductor pollicis longus tendon, and $1.5 \mathrm{~B}$-cun superior to the palmar wrist crease), Chize point (LU5; on the anterior aspect of the elbow, at the cubital crease, and in the depression lateral to the biceps brachii tendon), and Zusanli point (ST36; on the anterior aspect of the leg, on the line connecting ST35 with ST41, and 3 B-cun inferior to ST35) based on the conventional therapy (Figures 1-4). Dilatational wave $(2 / 50 \mathrm{~Hz})$ was selected with a wave width of $300 \mu \mathrm{s}$. The stimulus intensity was gradually increased from 0 with a gradient of $0.1 \mathrm{~mA}$, which caused sour, hemp, swelling, and heavy feelings, suggesting that "acupuncture brings about the desired sensation." [17] Then, the stimulus intensity was maintained at this level for continuous stimulation for $30 \mathrm{~min}$, and the treatment was administered once daily for 5 consecutive days. The treatment criteria of the three groups were based on the diagnosis and treatment guidelines for acute lung injury/ ARDS. The vital signs, CVP, $\mathrm{SaO} 2$, and urine of the patients were closely monitored $[18,19]$.

2.1. Data Collection. All the patients were included in the study after they were confirmed as having APALI in $24 \mathrm{~h}$. Arterial blood samples were drawn and arterial blood gas analysis indices were detected before (T1) and 3 (T2) and 5 (T3) days after electroacupuncture treatment using a GEM3000 blood gas analyzer for all three groups. The oxygenation index $(\mathrm{PaO} 2 / \mathrm{FiO} 2)$ was calculated for determining the APACHE-II scores. APACHE-II ("Acute Physiology and Chronic Health Evaluation II") is a severity-of-disease classification system, one of several ICU scoring systems. It is applied with 24 hours of admission of a patient to an intensive care unit (ICU): an integer score from 0 to 71 is computed based on several measurements; high scores correspond to more severe disease and a higher risk of death. Then, $5 \mathrm{~mL}$ of peripheral blood samples was collected from all three groups at $T 1$ and $T 3$. The blood samples were allowed to rest at room temperature for $30 \mathrm{~min}$ or at $4^{\circ} \mathrm{C}$ overnight, after which they were centrifuged at $2000 \mathrm{rpm} / \mathrm{min}$ for $20 \mathrm{~min}$ to obtain sera, which were collected and preserved at $20^{\circ} \mathrm{C}$. Fiber bronchoscopy and alveolar lavage were used to collect samples. Approximately 5-10 mL of bronchoalveolar lavage fluid (BALF) was collected. After centrifugation at $2000 \mathrm{rpm} / \mathrm{min}$ for $20 \mathrm{~min}$, the supernatant was collected and preserved at $20^{\circ} \mathrm{C}$, in order to avoid repeated freezing and thawing. An ELISA kit, purchased from Wuhan Youersheng (USCN) Technology Co., Ltd., was used to determine the TNF- $\alpha$ and IL-10 concentrations in the BALF and serum, according to the manufacturer's protocol. The primary observation indices were employed to calculate the oxygenation index $\left(\mathrm{PaO}_{2} /\right.$ $\mathrm{FiO}_{2}$ ). The secondary observation indices included the TNF$\alpha$ and IL-10 concentrations in the serum and BALF. The acute pathophysiology and chronic health assessment- (APACHE-) II score was used in the present study.

2.2. Statistical Analysis. SPSS 18.0 was used for statistical data analysis of the data obtained in this study. Data are shown as the number of subjects, mean \pm standard deviation, or mean \pm standard deviation of the mean. Comparisons between groups were performed using the $\chi^{2}$ test for categorical variables. The continuous response variables like age, weight, $\mathrm{PaO} 2 / \mathrm{FiO} 2$, and APACHE-II score are presented as mean \pm SD. One-way ANOVA was applied to compare the means among the 3 groups. Variables with repeated measures, such as arterial blood gas analysis parameters, were analyzed using repeated measures analysis of variance. $P<0.05$ was regarded as a significant difference. 


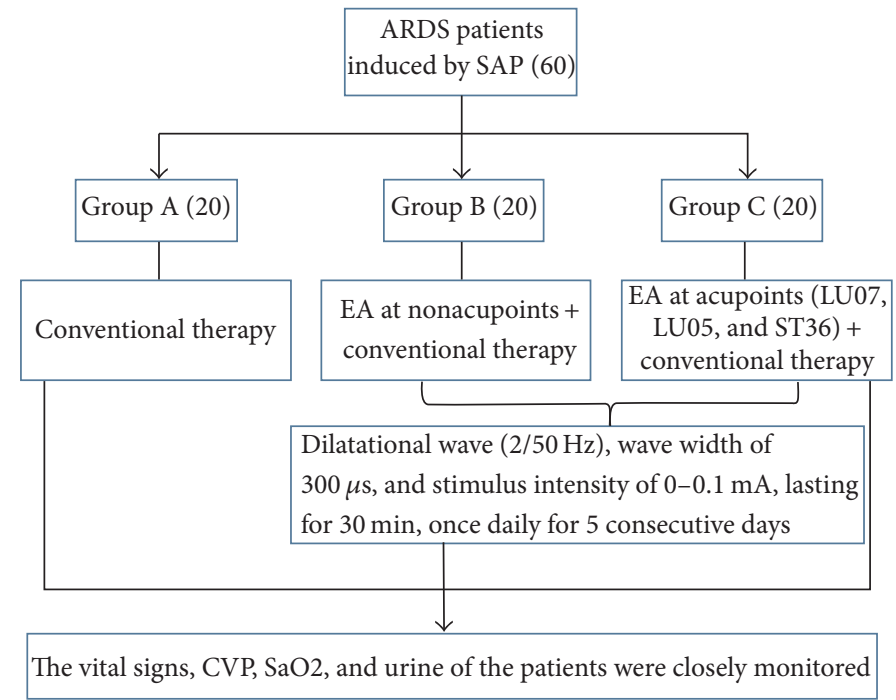

FIGURE 1: Flowchart about the group.

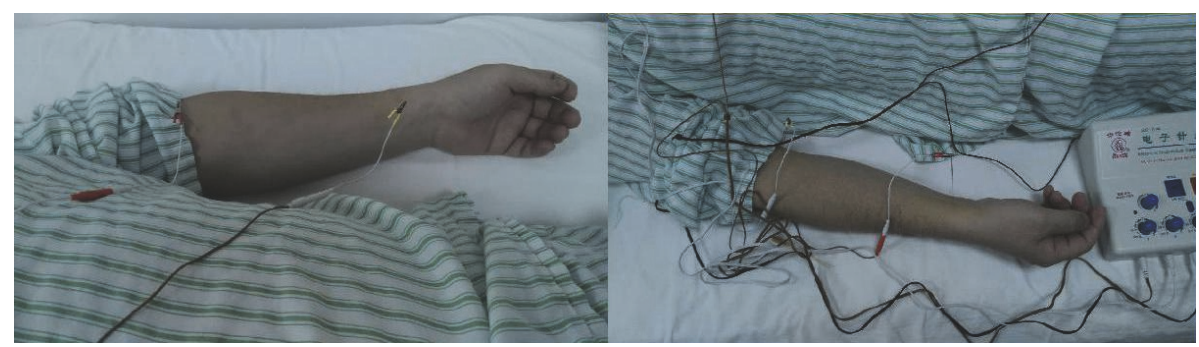

FigURE 2: Electroacupuncture was treated at Lieque (LU7) and Chize (LU5).

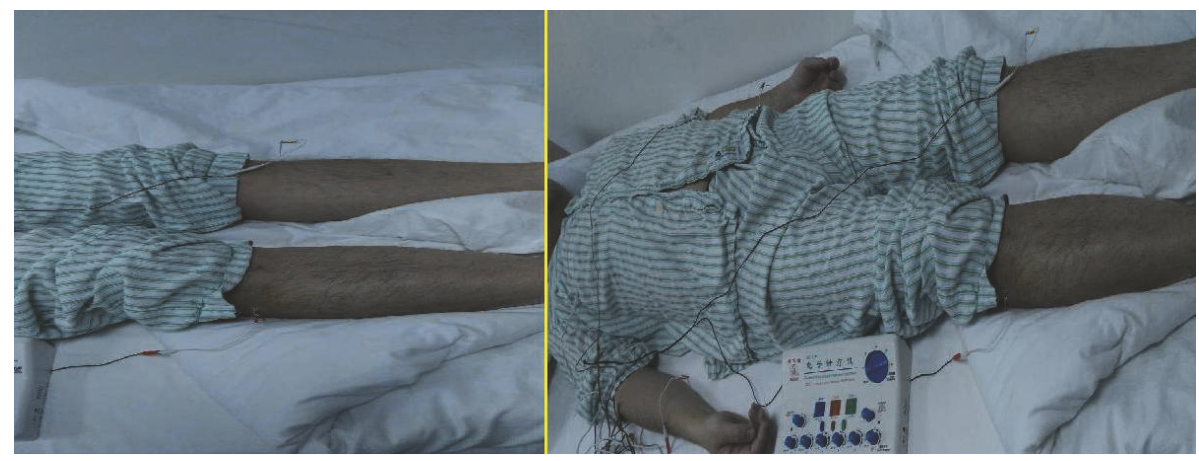

FIgURE 3: Electroacupuncture was treated at Zusanli (ST36).
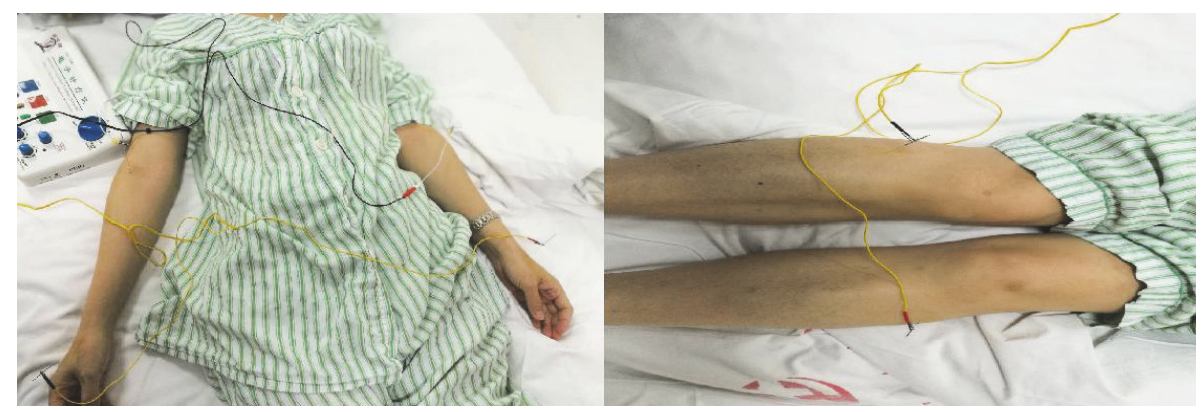

FIGURE 4: Electroacupuncture of nonacupoints (one centimeter away from Lieque, Chize, and Zusanli points but different from the traditional Chinese medicine "Jing Luo"). 
TABLE 1: Comparison of various general indicators in the three patient groups $(\bar{x} \pm S)$.

\begin{tabular}{lcccc}
\hline Indices & Group A & Group B & Group C & $P$ value \\
\hline Gender (cases, male/female) & $11 / 9$ & $12 / 8$ & $11 / 9$ & 0.934 \\
Aged (years) & $54 \pm 12$ & $58 \pm 12$ & $57 \pm 11$ & 0.5333 \\
Body weight (kg) & $67.5 \pm 10.5$ & $70.6 \pm 9.6$ & $62.6 \pm 13.6$ & 0.0894 \\
$\mathrm{PaO}_{2} / \mathrm{FiO}_{2}(\mathrm{mmHg})$ & $203.2 \pm 31.4$ & $215.2 \pm 42.7$ & $181.3 \pm 58.4$ & 0.0661 \\
$\mathrm{APACHE}_{\mathrm{II}}$ score & $17.9 \pm 3.3$ & $20.2 \pm 3.1$ & $19.3 \pm 3.7$ & 0.1038 \\
\hline
\end{tabular}

TABLE 2: Comparison of the parameters of groups A and B when there was a correlation between the groups $(\bar{x} \pm S)$.

\begin{tabular}{lccc}
\hline & Group A & Group B & $P$ value \\
\hline Difference in the oxygenation index $T_{2}-T_{1}$ & $16 \pm 4$ & $17 \pm 6$ & $36 \pm 5$ \\
Difference in the oxygenation index $T_{3}-T_{1}$ & $38 \pm 3$ & $1.5 \pm 1.2$ & 0.2694 \\
Difference in score $T_{1}-T_{2}$ & $1.4 \pm 2.8$ & $3.8 \pm 1.3$ & 0.0667 \\
Difference in score $T_{1}-T_{3}$ & $3.2 \pm 1.5$ & $3.6 \pm 1.9$ & 0.0922 \\
Plasma TNF- $\alpha T_{1}-T_{3}$ & $3.4 \pm 2.8$ & $5.1 \pm 2.8$ & 0.3965 \\
Plasma IL-10 T3-T1 & $4.8 \pm 2.9$ & $22.3 \pm 4.9$ & 0.3705 \\
BALF TNF- $\alpha T 1-T 3$ & $20.5 \pm 5.3$ & $6.1 \pm 2.4$ & 0.1359 \\
BALF IL-10 T3-T1 & $5.6 \pm 4.2$ & 0.3233 \\
\hline
\end{tabular}

\section{Results}

There were no significant differences in the gender constitution, age, body weight, and APACHE-II scores before treatment and the oxygenation index between the three groups showed also no significant difference $(P>0.05$; Table 1$)$. The oxygenation index in all groups at $T 2$ and $T 3$ was significantly improved compared with that at $T 1$ and APACHE-II scores were decreased $(P<0.05$; Tables 3 and 4$)$. Compared with group $\mathrm{A}$, the oxygenation index of group $\mathrm{C}$ increased at $\mathrm{T} 2$ and T3, and the APACHE-II score decreased at T3 $(P<0.05$; Table 3). In comparison, there was no significant difference in the above parameters in group $\mathrm{B}(P>0.05$; Table 2$)$. The serum and BALF concentrations of TNF- $\alpha$ in the three groups decreased at $T 3$ compared to $T 1$, while the concentration of IL-10 increased $(P<0.05$; Tables 3 and 4$)$. Compared with the values in group $\mathrm{A}$, the serum and BALF concentrations of TNF- $\alpha$ in group $C$ decreased at $T 3$ while the concentration of IL-10 increased $(P<0.05$; Table 3$)$. In comparison, there was no significant difference between the above parameters in group B $(P>0.05$; Table 2$)$. There were no significant differences in the side effects between the three groups $(P>$ 0.05; Table 5).

\section{Discussion}

TNF- $\alpha$ is a cytokine that is derived from a number of cells, but its main sources are the macrophages and monocytes. It interacts with a number of other cytokines such as IL-1, IL-6, and platelet activation factor (PAF) and has a pivotal role in the inflammatory response. It has a short plasma half-life, of 14-18 min, due to rapid clearance by the liver, gastrointestinal tract, and kidney, making its presence difficult to assess by serum assays. Therefore, absent or low levels of TNF in serum do not correlate well with actual events in the internal milieu.
While it is difficult to measure TNF levels in peripheral blood, certain studies have shown increased TNF levels in 30\%-40\% of patients with AP. In experimental studies, IL-10 has been shown to decrease levels of inflammatory markers and reduce the severity of pancreatitis. In experimentally induced pancreatitis, IL-10 levels parallel serum TNF levels and anti-IL-10 treatment reduce lung injury and pancreatic acinar necrosis, as well as reducing mortality from $42 \%$ to $0 \%$. Synthetic IL-10 agonist pretreatment reduced lung injury and mortality from experimental pancreatitis. The results of the present research illustrate that the level of the proinflammatory factor TNF- $\alpha$ decreased while the level of the anti-inflammatory cytokine IL-10 increased in patients with SAP after they received conventional therapy in combination with electroacupuncture at the Zusanli, Lieque, and Chize points, indicating that electroacupuncture at the three acupoints can flexibly promote the level of proinflammatory factors in patients with SAP and elevate the concentrations of anti-inflammatory factors, both of which are vital in the treatment of patients with SAP. Some scholars have found that preventive electroacupuncture at Zusanli could alleviate acute lung injury induced by lipopolysaccharide in rats, and the mechanism involved the inhibition of TNF- $\alpha$ transcription [20]. In addition, electroacupuncture at Zusanli exerted an antiinflammatory effect via the autonomic nervous system, attenuated the lipopolysaccharide-induced systemic inflammatory response, and improved the survival rate of rats with endotoxemia [21]. Both the Lieque and Chize points are important acupoints on the lung meridian, improving and treating respiratory system diseases and participating in the regulation of lung function. The electroacupuncture at the Lieque and Chize points through skin acupoints could regulate the autonomic nervous system, promote the release of endorphins in the hypothalamus, and inhibit the release of inflammatory factors and improve pulmonary function $[12,13]$. A previous 
TABLE 3: Comparison of the differences in parameters when there is correlation between groups A and C $(\bar{x} \pm S)$.

\begin{tabular}{lccc}
\hline & Group A & Group C & $P$ value \\
\hline Difference in the oxygenation index $T_{2}-T_{1}$ & $16 \pm 4$ & $48 \pm 8$ & $<5 \pm 9$ \\
Difference in the oxygenation index $T_{3}-T_{1}$ & $38 \pm 3$ & $2.8 \pm 1.8$ & $<0.0001$ \\
Difference in score $T_{1}-T_{2}$ & $1.4 \pm 2.8$ & $6.1 \pm 1.2$ & 0.0338 \\
Difference in score $T_{1}-T_{3}$ & $3.2 \pm 1.5$ & $12.1 \pm 3.5$ & $<0.0001$ \\
Plasma TNF- $\alpha T_{1}-T_{3}$ & $3.4 \pm 2.8$ & $13.7 \pm 3.8$ & $<0.0001$ \\
Plasma IL-10 $T_{3}-T_{1}$ & $4.8 \pm 2.9$ & $25.6 \pm 5.2$ & $<0.0001$ \\
BALF TNF- $\alpha T_{1}-T_{3}$ & $20.5 \pm 5.3$ & $22.8 \pm 5.9$ & 0.0020 \\
BALF IL-10 $T_{3}-T_{1}$ & $5.6 \pm 4.2$ & $<0.0001$ \\
\hline
\end{tabular}

TABLE 4: Comparison of the differences in parameters when there is correlation between groups B and C $(\bar{x} \pm S)$.

\begin{tabular}{lccc}
\hline & Group B & Group C & $P$ value \\
\hline Difference in the oxygenation index $T_{2}-T_{1}$ & $17 \pm 6$ & $48 \pm 8$ & $<0.0001$ \\
Difference in the oxygenation index $T_{3}-T_{1}$ & $36 \pm 5$ & $2.8 \pm 1.8$ & $<0.0001$ \\
Difference in score $T_{1}-T_{2}$ & $1.5 \pm 1.2$ & $6.1 \pm 1.2$ & 0.0338 \\
Difference in score $T_{1}-T_{3}$ & $3.8 \pm 1.3$ & $12.1 \pm 3.5$ & $<0.0001$ \\
Plasma TNF- $\alpha T_{1}-T_{3}$ & $3.6 \pm 1.9$ & $13.7 \pm 3.8$ & $<0.0001$ \\
Plasma IL-10 $T_{3}-T_{1}$ & $5.1 \pm 2.8$ & $25.6 \pm 5.2$ & $<0.0001$ \\
BALF TNF- $\alpha T_{1}-T_{3}$ & $22.3 \pm 4.9$ & $22.8 \pm 5.9$ & 0.0020 \\
BALF IL-10 $T_{3}-T_{1}$ & $6.1 \pm 2.4$ & & $<0.0001$ \\
\hline
\end{tabular}

TABLE 5: Comparison of the differences in side effects of three groups (\%).

\begin{tabular}{lccc}
\hline Group & Local pain & Local swollen & Local bleeding \\
\hline A & $2(10 \%)$ & $2(10 \%)$ & $1(5 \%)$ \\
B & $3(15 \%)$ & $3(15 \%)$ & $2(10 \%)$ \\
C & $2(10 \%)$ & $2(10 \%)$ & $1(5 \%)$ \\
$P$ value & 0.851 & 0.851 & 0.765 \\
\hline
\end{tabular}

study has shown a negative correlation between APACHEII scores and mortality of patients with ARDS [22]. The APACHE-II score is an important prognostic indicator for patients with ARDS. The results of the present research show that, in the patients with SAP who received conventional therapy combined with electroacupuncture at the Zusanli, Lieque, and Chize points, the oxygenation index was significantly higher, and the APACHE-II score was significantly decreased, suggesting that electroacupuncture at these three acupoints can effectively treat patients with acute lung injury [23]. The cause of death in patients with organ dysfunction is complicated and many interventions are required to treat these patients. In such cases, electroacupuncture can be used as an important measure to alleviate the lung injury induced by severe acute pancreatitis. The limitation of this study is its relatively small sample size. Because the current experiment is a single-center clinical trial, the number of patients who were admitted to this study is limited. Further research should aim to organize multicenter clinical studies based on the present study.

\section{Conclusions}

In summary, electroacupuncture at the Lieque, Chize, and Zusanli points can further improve the oxygenation index of patients with lung injury induced by SAP and alleviate the APALI, and the mechanism may be related to the downregulation of the TNF- $\alpha$ level and the upregulation of the IL-10 concentration.

\section{Conflicts of Interest}

The authors declare that they have no conflicts of interest.

\section{References}

[1] H. Ge, G. Yang, L. Huang, D. L. Motola, T. Pourbahrami, and C. Li, "Oligomerization and regulated proteolytic processing of angiopoietin-like protein 4," Journal of Biological Chemistry, vol. 279, no. 3, pp. 2038-2045, 2004.

[2] W. Zhao, W. Gong, and Y. Yang, "Research progress of angiogenesis like protein 2 and inflammation-related diseases," Chinese Journal of Pathophysiology, vol. 28, pp. 1986-1990, 2012.

[3] I. Kim, H.-G. Kim, H. Kim et al., "Hepatic expression, synthesis and secretion of a novel fibrinogen/angiopoietin-related protein that prevents endothelial-cell apoptosis," Biochemical Journal, vol. 346, no. 3, pp. 603-610, 2000.

[4] X. Lei, F. Shi, D. Basu et al., "Proteolytic processing of angiopoietin-like protein 4 by proprotein convertases modulates its inhibitory effects on lipoprotein lipase activity," The Journal of Biological Chemistry, vol. 286, no. 18, pp. 15747-15756, 2011.

[5] D. M. Valenzuela, J. A. Griffiths, and J. Rojas, "Angiopoietins 3 and 4: diverging gene counterparts in mice and humans," 
Proceedings of the National Academy of Sciences of the United States of America, vol. 96, no. 5, pp. 1904-1909, 1999.

[6] C. Suri, P. F. Jones, S. Patan et al., "Requisite role of angiopoietin1, a ligand for the TIE2 receptor, during embryonic angiogenesis," Cell, vol. 87, no. 7, pp. 1171-1180, 1996.

[7] Y. Y. Goh, M. Pal, H. C. Chong et al., "Angiopoietin-like 4 interacts with matrix proteins to modulate wound healing," Journal of Biological Chemistry, vol. 285, no. 43, pp. 32999-33009, 2010.

[8] A. Georgiadi, L. Lichtenstein, T. Degenhardt et al., "Induction of cardiac angptl 4 by dietary fatty acids is mediated by peroxisome proliferator-activated receptor $\beta / \delta$ and protects against fatty acid-induced oxidative stress," Circulation Research, vol.106, no. 11, pp. 1712-1721, 2010.

[9] G. Li, S. Li, L. An, and B. Wang, "Electroacupuncture alleviates intraoperative immunosuppression in patients undergoing supratentorial craniotomy," Acupuncture in Medicine, vol. 31, no. 1, pp. 51-56, 2013.

[10] G. Gu, Z. Zhang, G. Wang et al., "Effects of electroacupuncture pretreatment on inflammatory response and acute kidney injury in endotoxaemic rats," Journal of International Medical Research, vol. 39, no. 5, pp. 1783-1797, 2011.

[11] W. Gao, Y. Huang, H. Chen et al., "Regulatory effects of electro-acupuncture at Zusanli on brain-gut peptide contents in rats' pituitary gland and blood," Journal of the Fourth Military Medical University, vol. 22, pp. 793-796, 2001.

[12] K. S. L. Lau and A. Y. M. Jones, "A single session of Acu-TENS increases FEV1 and reduces dyspnoea in patients with chronic obstructive pulmonary disease: a randomised, placebo-controlled trial," Australian Journal of Physiotherapy, vol. 54, no. 3, pp. 179-184, 2008.

[13] S. P. C. Ngai, A. Y. M. Jones, C. W. Y. Hui-Chan, F. W. S. Ko, and D. S. C. Hui, "Effect of Acu-TENS on post-exercise expiratory lung volume in subjects with asthma-A randomized controlled trial," Respiratory Physiology and Neurobiology, vol. 167, no. 3, pp. 348-353, 2009.

[14] K.-A. Chu, Y.-C. Wu, Y.-M. Ting, H.-C. Wang, and J.-Y. Lu, "Acupuncture therapy results in immediate bronchodilating effect in asthma patients," Journal of the Chinese Medical Association, vol. 70, no. 7, pp. 265-268, 2007.

[15] S. Li, Z. Li, W. Yang, Y. Zhao, and J. Jie, "Comparative study of electroacupuncture at Shangjuxu (ST 37) and Chize (LU 5) on TNF-alpha content in the lung and colon and morphology of colonic mucosa in rats with ulcerative colitis," Global Chinese Medicine, pp. 415-419, 2014.

[16] V. M. Ranieri, G. D. Rubenfeld, B. T. Thompson et al., "Acute respiratory distress syndrome: the Berlin definition," The Journal of the American Medical Association, vol. 307, no. 23, pp. 25262533, 2012.

[17] J.-B. Yu, S.-A. Dong, L.-R. Gong et al., "Effect of electroacupuncture at Zusanli (ST36) and Sanyinjiao (SP6) acupoints on adrenocortical function in etomidate anesthesia patients," Medical Science Monitor, vol. 20, pp. 406-412, 2014.

[18] E. L. Bradley and N. D. Dexter, "Management of severe acute pancreatitis: A surgical odyssey," Annals of Surgery, vol. 251, no. 1, pp. 6-17, 2010.

[19] Pancreatic surgery Branch of Chinese Medical Association, "Guidelines for diagnosis and treatment of severe acute pancreatitis," Chinese Journal of surgery, vol. 45, no. 11, pp. 727-729, 2007.

[20] A. De Sá Ferreira, J. G. Mourão E Lima, T. P. Teixeira Ferreira, C. M. Tavares Lopes, and R. Meyer, "Prophylactic effects of short-term acupuncture on Zusanli (ST36) in Wistar rats with lipopolysaccharide-induced acute lung injury," Journal of Chinese Integrative Medicine, vol. 7, no. 10, pp. 969-975, 2009.

[21] J.-G. Song, H.-H. Li, Y.-F. Cao et al., "Electroacupuncture improves survival in rats with lethal endotoxemia via the autonomic nervous system," Anesthesiology, vol. 116, no. 2, pp. 406414, 2012.

[22] T. Luecke, E. Muench, H. Roth et al., "Predictors of mortality in ARDS patients referred to a tertiary care centre: A pilot study," European Journal of Anaesthesiology, vol. 23, no. 5, pp. 403-410, 2006.

[23] M. Fang, L. Zhang, D.-B. Wang et al., "Clinical analysis on changes of copeptin levels in serum and bronchoalveolar lavage fluid in patients with acute lung injury," Chinese Journal of Critical Care Medicine(Electronic Editon), no. 3, pp. 155-161, 2011. 


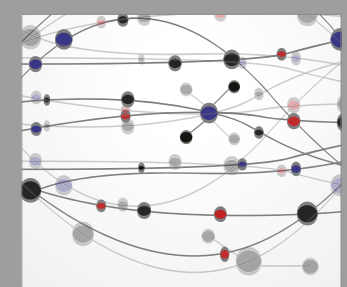

The Scientific World Journal
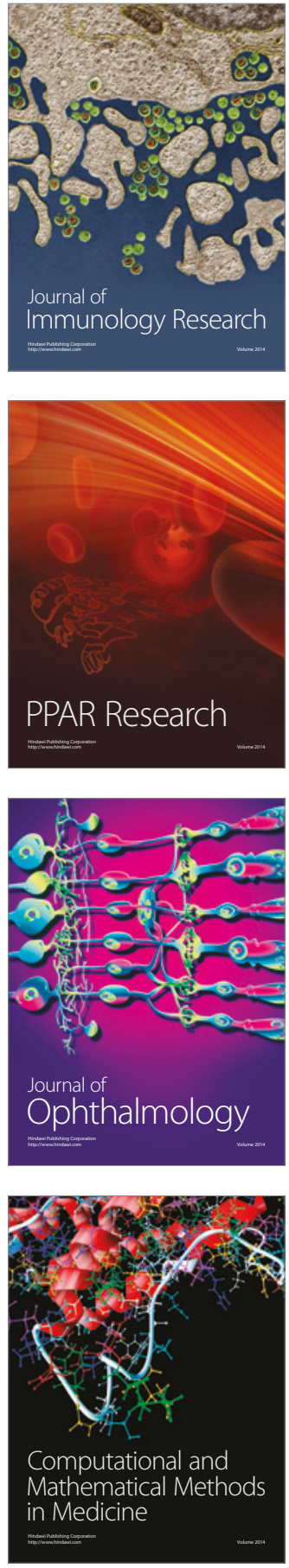

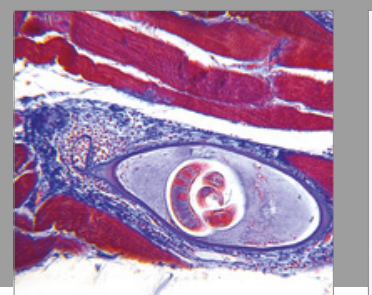

Gastroenterology Research and Practice
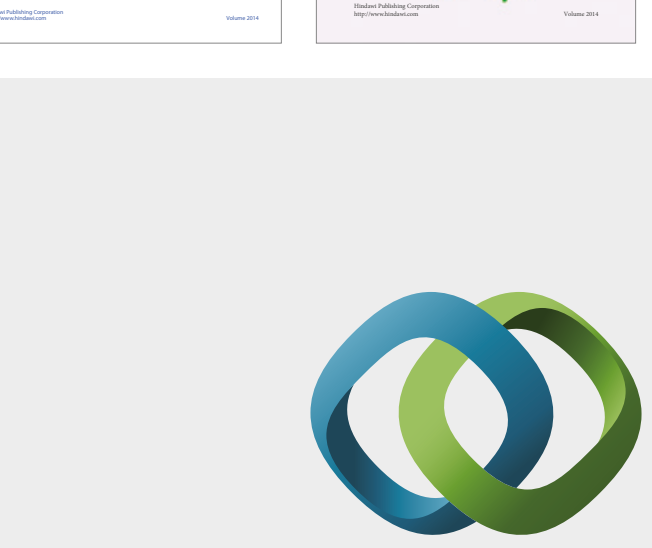

\section{Hindawi}

Submit your manuscripts at

https://www.hindawi.com
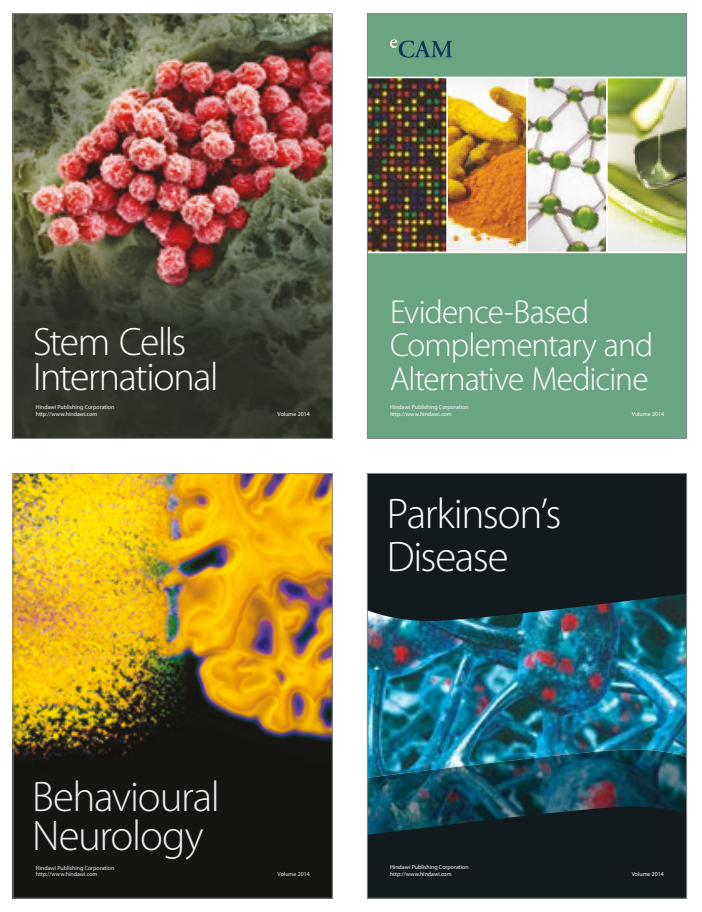
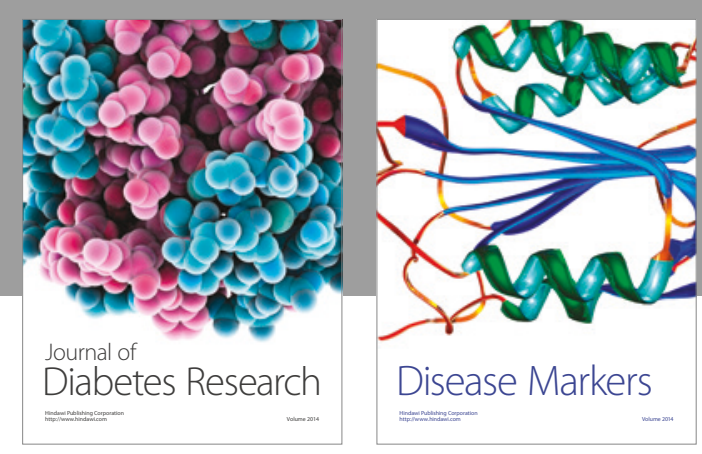

Disease Markers
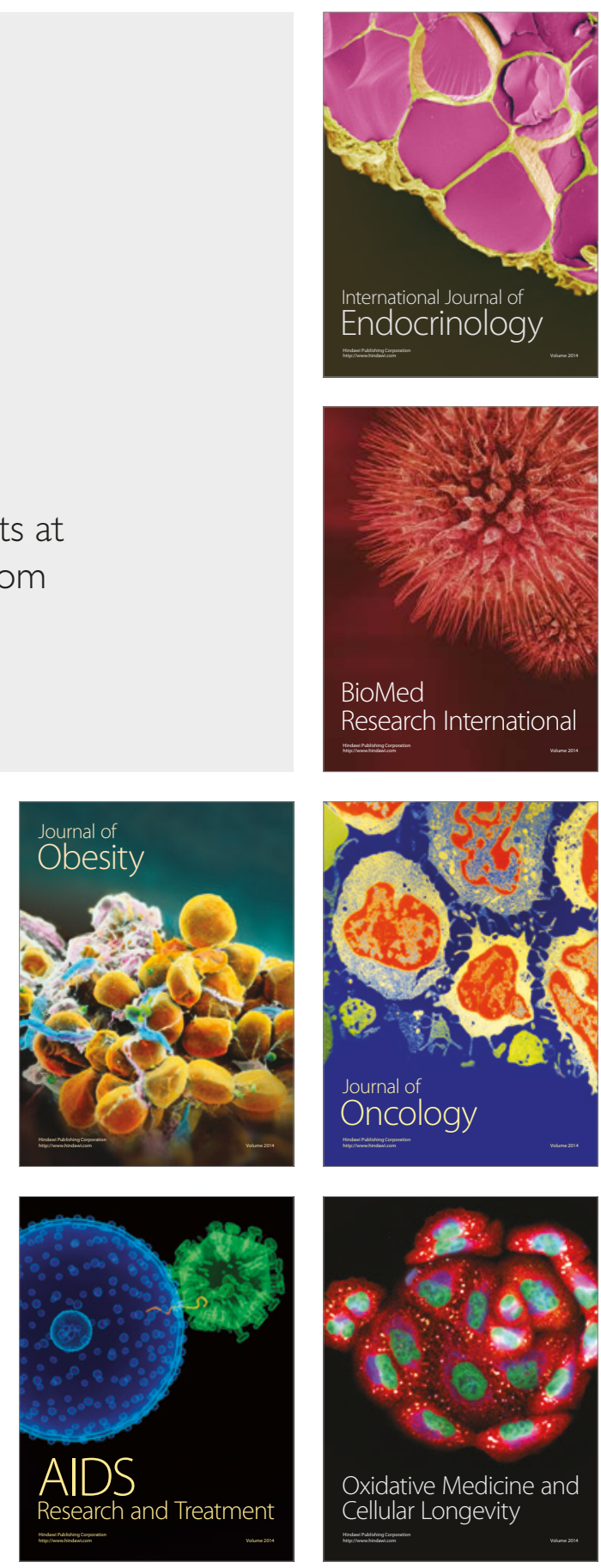